\section{Direct Reprobing with Anti- $\beta$-actin Antibody as an Internal Control for Western Blotting Analysis}

BioTechniques 28:216-218(February 2000)

Western blotting analysis is the most common method for identification and quantification of a specific protein in complex protein mixtures (2-4). To compare different levels of an interesting protein derived from different sources, such as from different tissues or from cell cultures exposed to a variety of treatments, an internal control is necessary to ensure even sample loading and gel transfer. Internal controls normally are housekeeping gene products because their expression levels are the same in most tissues and are presumably not affected by most treatments. Among these, $\beta$-actin is one of the most common proteins used for this purpose.

There are at least two methods for performing the internal control analysis. The membrane that is used to detect the target protein is stripped and reprobed by another antibody against the internal control protein. This procedure alone could take two days. Also, the stripping procedure tends to elute proteins from blots that result in progressively decreasing signal intensities, and the binding affinities of proteins to membrane and/or antibodies to target proteins can be different, which results in poor reliability. Occasionally, duplicate membranes, one for detecting the target molecule and one for the internal control analysis, are generated under identical conditions. However, this strategy is more costly and still does not eliminate possible pipetting and transferring errors.

We found a simple method to perform the internal control analysis in which the blot is directly reprobed by the internal control antibody after detection of the primary target. The major idea for this method is based on the theory that the binding sites of the target protein and its primary antibody are saturated, and horseradish peroxidase (HRP) conjugated on secondary antibody has been inactivated totally by hydrogen peroxide $\left(\mathrm{H}_{2} \mathrm{O}_{2}\right)$ in substrate during the first detection (1). This method essentially avoids protein loss from the harsh stripping. Therefore, the detected internal control signal truly reflects protein loading.

In our experiment, cyclooxygenase 1 (Cox 1) was the target protein, and $\beta$ actin was used as an internal control. Thirty micrograms of total lysate of rat liver tissue from different treatments were separated on a $7.5 \%$ SDS-PAGE gel, then transferred to a piece of 0.2 $\mu \mathrm{m}$ nitrocellulose membrane (Bio-Rad Laboratories, Hercules, CA, USA). This membrane was blocked with blocking solution (5\% non-fat milk, $0.05 \%$ Tween ${ }^{\circledR} 20$ in PBS) overnight at $4^{\circ} \mathrm{C}$ with gentle shaking. The following morning, this membrane was rinsed once with PBST $(0.05 \%$ Tween 20 in phosphate-buffered saline), then incubated with goat anti-rat Cox 1 M-20 polyclonal antibody (Santa Cruz Biotechnology, Santa Cruz, CA, USA) with a dilution of 1:500 in blocking solution. This incubation was performed at room temperature for one hour with gentle rolling in a hybridization tube. After the primary antibody incubation, the membrane was rinsed twice with PBST, followed by two $5 \mathrm{~min}$ washes and two $10 \mathrm{~min}$ washes to eliminate nonspecific binding. The membrane was then incubated at room temperature for one hour with 1:2000 dilution of donkey anti-goat IgG secondary antibody conjugated with HRP (Santa Cruz Biotechnology). The wash step was as described before, except an additional 10 min wash was added. After incubating with SuperSignal ${ }^{\circledR}$ West Pico Chemiluminescent Substrate (Pierce Chemical, Rockford, IL, USA) at room temperature for $5 \mathrm{~min}$, the membrane was wrapped with a piece of plastic and exposed to an X-ray film (Eastman Kodak, Rochester, NY, USA) for imaging.

The Cox 1 blot membrane was kept at room temperature within the plastic wrap overnight. It was removed from the plastic wrap the next morning and washed once with PBST for 10 min at room temperature, then reprobed with a mouse monoclonal anti- $\beta$-actin anti-

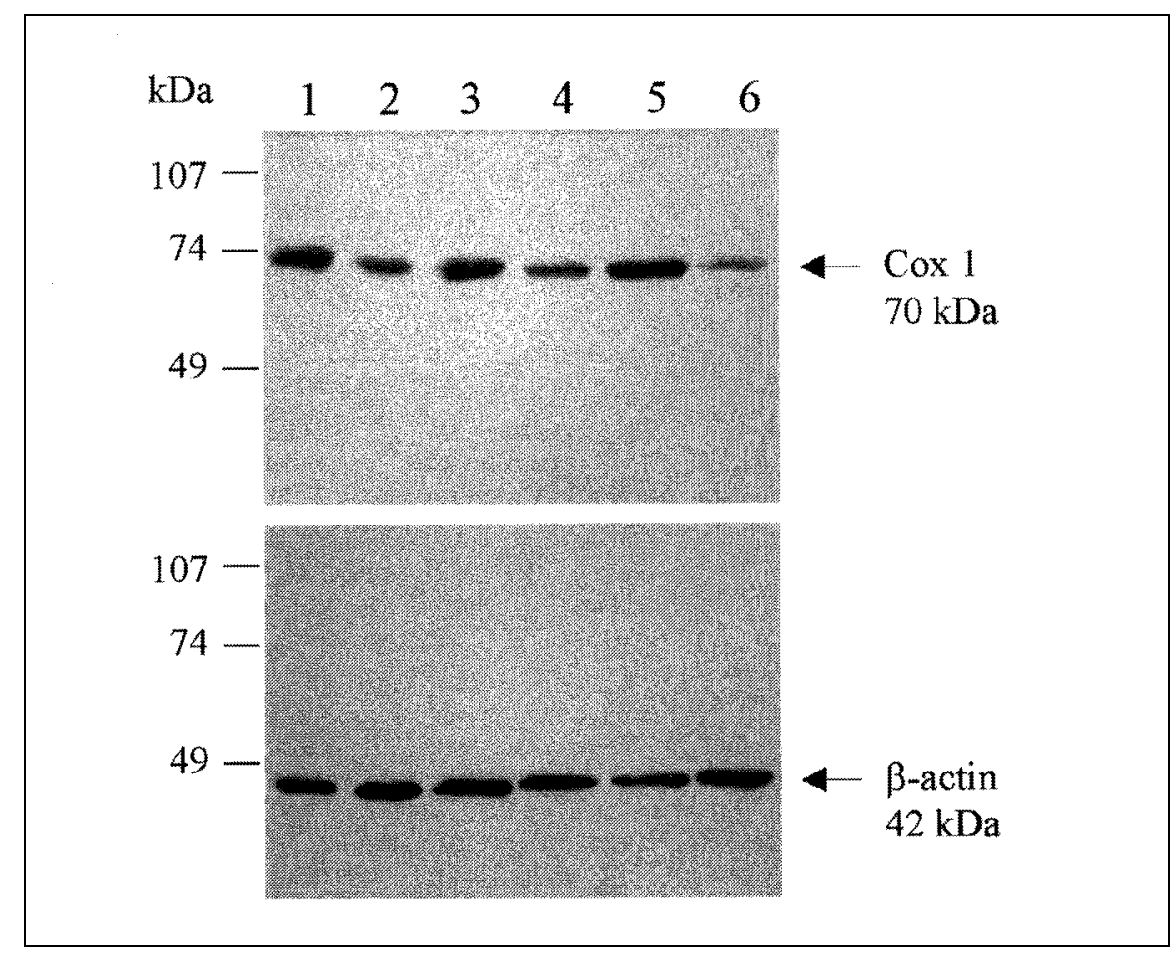

Figure 1. Western blotting analysis for Cox 1 and $\beta$-actin. Thirty micrograms of total lysate of rat liver tissue from untreated group (lanes 1,3 and 5) and treated group (lanes 2, 4 and 6) were resolved in a $7.5 \%$ SDS-PAGE gel and then transferred to a nitrocellulose membrane. The membrane was probed by M-20, a goat polyclonal anti-rat Cox 1 antibody (upper panel). The same membrane was directly reprobed by a monoclonal anti- $\beta$-actin antibody (lower panel). Arrows indicate the expected signals of Cox 1 and $\beta$-actin. 
body (Clone AC-150; Sigma, St. Louis, MO, USA) with a dilution of 1:5000 in blocking solution as previously described. The secondary antibody used for $\beta$-actin was HRP-conjugated goat polyclonal anti-mouse IgG (Transduction Laboratories, Lexington, KY, USA). The rest of the procedure was the same as for Cox 1.

Figure 1 shows that both Western blots were able to detect one single band with the expected molecular weight. As we expected, there was no detection of Cox 1 signal on the $\beta$-actin blot, even after a longer exposure (data not shown). This result is because the HRP on the Cox 1 blot had already been inactivated by hydrogen peroxide in SuperSignal substrate and the duration of chemiluminescence produced by SuperSignal substrate was only $6-8$ h. To further investigate the HRP inactivation rate and chemiluminescence duration, we then produced another Cox 1 blot. This one was exposed to an $\mathrm{X}$-ray film for $5 \mathrm{~min}$ immediately, and at 1,2 or $4 \mathrm{~h}$ after substrate incubation. We were not able to detect any signal at the $4 \mathrm{~h}$ time point (data not shown). This blot was then washed once with PBST for $10 \mathrm{~min}$ at room temperature and incubated with fresh substrate again for $5 \mathrm{~min}$. We did not detect any signal even after overnight exposure (data not shown), which indicates that the amount of hydrogen peroxide in SuperSignal substrate is sufficient to inactivate HRP $4 \mathrm{~h}$ after incubation. The following morning, this blot was washed once in PBST for $10 \mathrm{~min}$, and then the same procedure for Cox $1 \mathrm{de}$ tection was repeated. We failed to detect any Cox 1 signal even after overnight exposure (data not shown), indicating that normal washes in PBST are not sufficient to remove antibodies from an antigen-antibody complex.

For Western blotting analysis, the primary antibody is usually present at a much higher quantity than the target protein, as is the secondary antibody to primary antibody. Because the bound antibodies cannot be removed by normal washing, the antibodies directly reprobed for internal control will neither affect nor be affected by the detection of the primary target, even though the same secondary antibody is used. The direct reprobing method was also successfully used for detecting both $\beta$ actin and other target proteins, such as the HA epitope (antibody generated in rat) and the ErbB 4 (antibody generated in rabbit) from cultured cell lines (data not shown).

In summary, the entire procedure of the direct reprobing method for internal control analysis omits the stripping and reblocking steps. By using this method, signal attenuation by high-stringency stripping does not occur. Most importantly, the detected signals are more accurate for loading controls because proteins on the membrane are not eluted by stripping. We conclude that this direct reprobing method is a simple, inexpensive and accurate method for internal control in Western blotting analysis.

\section{REFERENCES}

1.Arnao, M.B., M. Acosta, J.A. del Río and F. García-Cánovas. 1990. Inactivation of peroxidase by hydrogen peroxide and its protection by a reductant agent. Biochim. Biophys. Acta, 1038:85-89.

2.Burnette, W.N. 1981. "Western blotting": electrophoretic transfer of proteins from sodium dodecyl sulfate-polyacrylamide gels to unmodified nitrocellulose and radiographic detection with antibody and radioiodinated protein A. Anal. Biochem. 112:195-203.

3.Fido, R.J., A.S. Tatham and P.R. Shewry. 1995. Western blotting analysis. Methods Mol. Biol. 49:423-437.

4.Towbin, H., T. Staehelin and J. Gordon. 1979. Electrophoretic transfer of proteins from polyacrylamide gels to nitrocellulose sheets: procedure and some applications. Proc. Natl. Acad. Sci. USA 76:4350-4254.

We thank Cynthia J. Woods and Destiny M. Hollis for critical proofreading. This work was supported by Grant No. 19020K120 to M.J.W. from the American Cancer Society. Address correspondence to Dr. Ji Liao, Department of Pathology, School of Medicine, University of South Carolina, 6439 Garners Ferry Road, Columbia, SC 29209 USA. Internet: jliao@med.sc.edu

Received 21 July 1999; accepted 5 November 1999.
Ji Liao, Xingzhi Xu and
Michael J. Wargovich
University of South Carolina
Columbia, SC, USA

\section{Lipopolysaccharide Affin- ity Measurement by Scin- tillation Proximity Assay: Application to Human Heparin Binding Protein}

\author{
BioTechniques 28:218-222 (February 2000)
}

Bacterial lipopolysaccharide (LPS, endotoxin), the principal lipid component of the outer leaflet of the outer membrane of Gram-negative bacteria, is recognized by defensive cells in essentially all multicellular organisms. Leukocytes respond to LPS by secreting a variety of cytokines. Although the secretory responses to LPS are usually beneficial, an exaggerated response may be fatal. Therefore, great efforts have been put into the development of assays suitable to assess LPS binding to its different targets, e.g., its cellular receptor and a variety of LPS binding proteins in the body.

A number of different cell-binding assays have been developed $(4,7)$. The majority of these are designed as separation assays, in which the cells of interest are incubated with labeled LPS and separated by centrifugation from the reaction mixture, after appropriate binding has taken place and following separation and washes. Cell-associated LPS is subsequently detected by means of liquid scintillation counting or fluorescence quantification (radio- and fluorescent probe-labeled ligands, respectively). However, in other situations, it might be of interest not to measure LPS binding to cells but to the purified components of the system. Previously, this has proven to be difficult.

Here, we present a homogeneous LPS binding assay based on scintillation proximity assay (SPA) technology. The purpose of the assay is to measure LPS affinity to any protein of interest.

In our laboratory, we wanted to investigate the LPS binding properties of recombinant human Heparin Binding Protein (hHBP, CAP37, azurocidin). $\mathrm{hHBP}$ is a neutrophil glycoprotein $(1,2)$ of importance in host defense during infections and inflammations (5). To obtain direct binding data, it was necessary to develop an assay that was not based 